\title{
Dictature militaire et université
}

\author{
Politiques, réactions et mémoires au Brésil
}

(1964-1985)

Roberta Borrione

(Docteure en histoire à l'EHESS)

\section{Résumé/Abstract}

[Fr] De 1964 à 1985, le Brésil vit une période de gouvernement militaire de type dictatorial. La politique intérieure vise surtout à justifier les actions gouvernementales et met en avant le rôle des Forces armées comme défenseur de la démocratie et des idéaux chrétiens face à l'invasion communiste et à la corruption. Tout un ensemble de politiques est mis en place, y compris au sein du système éducatif. La communauté universitaire réagit de manière hétérogène. Trente ans après la fin de la dictature, quatorze professeurs de l'Université de São Paulo sont interviewés. Leurs remémorations des faits montrent des sentiments et des affects différents, parfois contradictoires. Cinq types de parcours de vie se dessinent. Le premier tient à une résistance de type proprement universitaire. Il s'agit, sur place, en poste, d'assurer la continuité de la recherche et de l'enseignement. Un deuxième type de parcours consiste à trouver refuge dans la clandestinité pour contrecarrer l'idéologie militaire, en poursuivant ses activités du quotidien en toute légalité. La duplicité en constitue le ressort. Un troisième représente l'exil, soit à l'intérieur du Brésil, soit à l'étranger. Un quatrième type de parcours relève d'un calcul que dénoncent certains professeurs : mettre à profit le système de contrôle idéologique en place pour en tirer des profits personnels. Un cinquième consiste à refuser certains événements introduits par le nouveau régime. La vie continue, ignorant les changements sociopolitiques. Une fois la dictature affaiblie, les discussions intellectuelles reprennent leur ancien élan. Certains types de parcours de vie convergent même vers des actions dans la vie politique. Une mémoire universitaire se construit par la mise en valeur d'actes de bravoure universitaire en dépit des actes de répression militaire.

Mots-clés : dictature militaire, Université, mémoire, politiques, parcours de vie.

[En] From 1964 to 1985 , Brazil lives a period of dictatorial military government. Internal politics aim mostly to justify governmental actions and highlight the military forces as promoters of democracy and Christian ideals against corruption and communist invasion. A complex set of politics is put in place, including within the educational system. The university community reacts in different ways. Thirty years after the end of the Brazilian dictatorship, fourteen professors of the University of São 
Paulo were interviewed. Their memories of the facts show different feelings and emotions, at times contradictory. Five life pathways are drawn. The first one consists in remaining in the university to preserve research and teaching as an act of resistance. A second type of life pathway also consists in remaining in the university, yet at the same time offering clandestine resistance to the military ideology. This duplicity of action is the taken resort. A third type of life pathway refers to going into exile, either to different regions of the country, either abroad, as a life choice. A forth life pathway may be inferred by what some of the interviewees told: certain members of the university community used the ideological system of control to take personal advantages. And finally, the fifth life pathway consists in denying the social changes introduced by the new political system, life going on as if nothing had changed. Once the dictatorship began to fall, intellectual discussions are retaken. Some of the aforementioned life pathways converge toward political action. A university memory is built through the importance given to acts of bravery in spite of acts of military repression.

Keywords: military dictatorship, university, memory, politics, life pathways.

\section{Introduction}

Les décennies 1950 et 1960 se caractérisent par une forte agitation et par des conflits sociaux, économiques et politiques à l'échelle mondiale. Le Brésil ne fait pas exception à cette règle. Le 31 mars 1964, une dictature militaire, ou bien une dictature civile-militaire, s'instaure et perdure jusqu'en $1985^{1}$.

Dès les premiers jours au pouvoir, les militaires édictent plusieurs actes législatifs ayant, parmi d'autres missions, celles de légitimer leurs actions et de construire un imaginaire collectif sur le rôle et l'importance des Forces armées dans le gouvernement du pays. Ils établissent un complexe système de contrôle fondé sur l'espionnage ; la police politique ; la censure (de la presse et des loisirs) ; la propagande politique ; et les jugements arbitraires d'individus prétendus corrompus (Fico 2004 ; Magalhães 1997).

La communauté universitaire n'échappe pas aux politiques du gouvernement militaire. Comment cette communauté et plus particulièrement celle de l'Université de São Paulo (USP) vit-elle alors cette période de l'histoire brésilienne ? Comment la remémore-t-elle?

A partir d'une thèse doctorale, cet article se base sur les entretiens récoltés en 2006 auprès de quatorze professeurs de l'USP. Le but a consisté à reconstituer un quotidien universitaire marqué, bien évidemment, par des politiques et des actes

${ }^{1}$ Pour une discussion au sujet des dictatures militaires au Brésil et en Amérique Latine, cf. Coggiola 2001 ; Fausto 2001 ; Ferreira \& Delgado 2003 ; Santos 1994 ; Wasserman\&Guazzelli 2004. 
(universitaires et gouvernementaux) et par les réactions de sa communauté. On a aussi envisagé de dessiner les parcours de vie des acteurs universitaires et de mettre en lumière les mémoires et les significations construites au sujet de ce quotidien-là. Il s'agissait d'appréhender une histoire passée par le croisement des souvenirs de différents individus.

Sur quarante professeurs de l'USP contactés, une vingtaine d'entre eux ont répondu à l'appel, certains défavorablement. Avec le restant, il fut impossible de trouver un rendez-vous avant la fin du séjour à São Paulo. La fin de l'année universitaire avec ses contraintes d'évaluation et de remise de notes, la proximité avec les fêtes de fin d'année et les vacances scolaires furent des arguments utilisés pour expliquer cette difficulté. Les difficultés rencontrées pour contacter les professeurs, de même que la multitude d'arguments employés pour ne pas participer à l'étude, laissent présager d'un traumatisme encore très présent à témoigner de cette époque.

Entre 1964 et1985, sept interviewés étaient professeurs et les sept autres étudiants, parmi lesquels cinq ont également intégré le corps enseignant de l'USP durant la période. Il s'agit de sept femmes et sept hommes. Hormis une jeune professeure dans la quarantaine, les treize autres professeurs étaient sexagénaires, voire septuagénaires. Certains professeurs avaient déjà pris leur retraite et d'autres allaient le faire dans les mois suivants. Assurément, les interviewés ne sont pas à proprement parler représentatifs, ni de São Paulo, ni a fortiori du Brésil. Leurs capacités intellectuelles, leur organisation en associations, leurs traditions d'autonomie, sinon de critique, en font un secteur à part de la société urbaine. Ils et elles constituent des témoins privilégiés des traces laissées dans le temps social d'un système militaire à caractère dictatorial.

La date, l'horaire et le lieu de l'entretien furent laissés à la convenance des interviewées. Ils se sont donc déroulés dans leur maison ou sur leur lieu de travail (bureau à l'Université ou ailleurs), dans un espace qui leur était familier et dans un climat décontracté. Au début de l'entretien, les professeurs étaient une nouvelle fois informés que l'étude visait à analyser l'évolution du système éducatif brésilien, notamment de l'enseignement supérieur durant l'époque de la dictature militaire, et qu'elle concernait d'éventuelles influences sur les parcours de vie de membres de la communauté universitaire. Ensuite, il leur était demandé de raconter leur parcours acadêmico $^{2}$. Il ne leur était pas précisé une date ou une période spécifique. Certains sont remontés à l'époque de leur enfance, alors que d'autres ont commencé leur récit à partir de l'époque de la dictature militaire.

Dans le cadre d'entretiens semi-directifs, trois thèmes généraux orientaient mes interventions : les parcours de vie des interviewés ; la réforme de l'enseignement su-

\footnotetext{
${ }^{2}$ En portugais, le mot acadêmico fait allusion surtout à la formation universitaire et à la vie professionnelle universitaire, mais il laisse une petite marge de sens permettant aux individus de raconter également leur formation scolaire.
} 
périeur ; les politiques et les actions du gouvernement militaire sur le quotidien universitaire, précisément les mécanismes de contrôle de la circulation de l'information et des personnes; et ce, de manière à ce qu'ils et elles se remémorent l'époque et donnent leur interprétation, leur signification et leur évaluation des faits.

\section{Dictature faisant, des parcours de vie se dessinent}

Le récit des interviewés met en scène leurs sentiments et leurs affects. Leurs parcours individuels révèlent des similitudes face aux mêmes évènements, aux mêmes lois et permettent de construire un quotidien universitaire et des types de parcours de vie caractérisant cette période de l'histoire brésilienne, et cela selon cinq modalités distinctes. Différents par leurs ressorts, ils ont en commun de témoigner, trois fois sur cinq, d'un même refus de la dictature militaire.

\subsection{Résistance intellectuelle : la défiance ou l'autocensure ?}

Malgré les interdictions et les limites tacitement ou réellement imposées par le gouvernement militaire et par l'Université, la plupart des membres de la communauté universitaire et plus particulièrement les professeurs décident de continuer leurs travaux universitaires. Ils approuvent le fait de rester à l'Université. Continuer à développer leurs travaux de recherche et à donner des cours constitue une forme de résistance et ce, d'après ce que relatent les interviewés, surtout en raison de l'évaluation que certains professeurs, les plus anciens et les plus respectés, ont fait du contexte historique de l'époque. Ces derniers prévoyaient, ou bien espéraient, la fin de la dictature militaire. Ils conseillaient donc à leurs protégés, eux aussi professeurs d'université, de poursuivre les travaux.

Partageant un objectif commun, celui de préserver l'université et de la restituer « saine et sauve » à la société lors de la fin de la dictature militaire, les professeurs adoptent dans ce cadre des comportements différents, voire même divergents : défier le régime militaire par le travail intellectuel ou soumettre son travail à une autocensure?

Pour ce qui concerne la défiance intellectuelle, on raconte qu'il ne fallait pas accepter les injonctions gouvernementales en matière d'enseignement. Malgré la présence d'espions dans les classes et la peur d'être persécuté, le maintien de la bibliographie représentait un acte de résistance. La participation à des mouvements contre la dictature militaire en constituait un autre.

Les interviewés racontent leur participation à une lutte institutionnelle avec un grand sentiment de fierté. Ils ajoutent qu'il fallait garder la liberté d'expression universitaire ou, du moins, essayer de le faire. Une interviewée relate même que les militaires comparaient les professeurs à des trafiquants de drogues. C'est pourquoi il leur fallait les surveiller, eux les «trafiquants d'idées ». Nombreux sont les récits de 
cas, normalement isolés et parfois anecdotiques, qui mettent en avant la bravoure de ceux qui se sont exposés en montrant, par leurs paroles et souvent lors des cours, leur mécontentement à l'égard du gouvernement militaire.

En lien avec les phénomènes d'autocensure, on mettra en évidence le fait qu'occuper l'espace universitaire signifie parfois se mettre en retrait et limiter volontairement la portée de son travail. La fin de l'année 1968 semble avoir été décisive pour ce qui concerne l'adoption de ce type de comportement. Un interviewé déclare qu'il s'agissait d'un « accord tacite », même «plus que tacite », car soutenu par certains intellectuels. La communauté universitaire sait que les militaires fréquentent les cours à l'université et craint des représailles. C'est pourquoi ils limitent la bibliographie de leurs cours et témoignent de prudence lors de nouvelles rencontres.

Les comportements d'autocensure se limitaient parfois à des situations et à des sujets spécifiques. Ils semblent cependant faire partie du parcours de certains interviewés d'une manière beaucoup plus flagrante qu'on aurait pu l'imaginer. L'interviewé susmentionné se plaint que l'autocensure - en tant qu'engagement institutionnel, comme il le caractérise - n'est toujours pas reconnu par toute la communauté universitaire. Alors que les malaises endurés par les professeurs destitués font l'objet de nombreuses discussions jusqu'à nos jours et que les comportements de défiance sont exaltés, signale-t-il, on oublie les efforts des professeurs restés à l'université et ayant opté pour le retrait, afin de préserver l'espace académique et par-là le patrimoine culturel universitaire. Dans ce cas de figure, le professeur peut affirmer avoir adopté un tel comportement car il a été persécuté et mis sous surveillance militaire.

L'analyse des récits collectés montre qu'il est presque impossible d'avouer ou même de percevoir qu'on s'est soumis soi-même à un tel comportement, d'autant plus si la répression ne les touche pas directement. Parfois, c'est au moment de l'entretien qu'il apparaît sous la forme d'un questionnement: mon travail a-t-il été affecté par le contrôle idéologique du gouvernement militaire ? Mon comportement a-t-il changé face à ce contrôle ? Ici, les interviewés s'expriment de manière beaucoup moins volubile. Ils hésitent davantage. L'autocensure est un comportement que l'on réserve aux autres. On le comprend et on le justifie en signalant l'existence d'espions et de persécutions. Et quand on l'avoue et on le perçoit pour soi-même, on le fait en disant que toute la communauté universitaire s'était imposée une autocensure.

\subsection{Militantisme et dictature : la solution d'une double vie}

Historiquement, il est avéré que le contrôle du gouvernement militaire avait réussi à pénétrer le milieu universitaire. Face à cette situation, une partie de la communauté universitaire a opté de ne pas l'affronter ouvertement. Certains individus choisissent d'éviter les mesures de contrôle, sans pour autant les accepter. 
Vie professionnelle et vie privée divergent. Leur duplicité les incite à mettre en avant certaines caractéristiques socialement acceptées et à dissimuler certains aspects de leur vie personnelle et professionnelle, plus particulièrement les parties soidisant « subversives ».

À l'époque, on cachait les actions militantes, qu'elles aient été exercées dans le champ de l'intellectualité, de la politique, de la clandestinité, tout autant que de la lutte armée. Lors des entretiens, les interviewés profitent de leur parole pour énumérer, expliquer et donner une photographie, sinon exacte du moins colorée, des activités clandestines. Les sujets de discussion à l'université diffèrent de ceux débattus en privé, racontent en souriant certains interviewés. Certains professeurs et étudiants cherchent également à contourner la censure imposée sur certaines informations. Cela se réalise par l'organisation de réunions clandestines, par la réalisation de sketchs dans des lieux publics ou encore, notamment, par l'édition et la distribution de pamphlets comportant des informations censurées. L'affiliation à un parti politique clandestin ainsi que toute activité qui en découlait étaient dissimulées.

Le militantisme par la lutte armée figure comme un parcours d'exception chez les professeurs et chez les étudiants. Aujourd'hui encore, il reste difficile à leurs auteurs d'en parler. De fait, aucune et aucun interviewé ne déclare s'y être engagé. Au contraire, ils précisent même qu'ils évitaient de recourir à la lutte armée. C'est uniquement l'une d'entre eux qui remémore un tel parcours. Elle ne dit pas ouvertement avoir fait partie du militantisme armé. Cependant, son discours - ses mots, ses silences, ses affects, ses jugements et ses explications - le dénonce.

Mener une double ou une triple vie n'a pas empêché que le régime militaire ne retrouve les traces d'un militantisme. Toutefois, les poursuites diffèrent selon les cas individuels. En tout cas, le refus de se soumettre est manifeste, quelles qu'en aient été les modalités et la mémoire des interviewés, plusieurs décennies après. Les interviewés tirent une certaine fierté d'avoir des dimensions officielles et clandestines de leur vie. Leurs récits exaltent leur intelligence et leurs astuces pour détourner les contrôles militaires, les risques de certaines activités et leurs sentiments positifs de faire partie d'une telle lutte.

\subsection{L'exil face à l'oppression dictatoriale}

Hormis quelques cas, le gouvernement dictatorial brésilien n'impose pas l'exil à ses citoyens. Ceci dit, un troisième type de parcours de vie a été celui de l'exil. Face aux actes de contrôle idéologique et de répression du gouvernement militaire, un certain nombre de Brésiliens, issus d'une grande diversité de milieux, ont opté pour l'exil.

Ici, le terme « exil » n'a pas sa signification stricte d'expulsion de quelqu'un hors de sa patrie. Une signification plus large lui est accordée. Elle constitue le fait de se sentir forcé à séjourner hors de son lieu d'origine. Quitter soit son pays, soit sa région, son État ou sa ville, ce choix se faisant surtout en fonction de la notoriété de l'individu en question. Qui connaît une reconnaissance nationale finit par quitter le 
Brésil pour les pays d'Europe, d'Amérique du Nord, à moins qu'il reste au Sud. Toutefois, les coups d'État successifs dans les pays sud-américains engendrent une succession d'exils. Rester dans le pays a constitué le choix de jeunes intellectuels et de ceux dont la notoriété était locale et restreinte. Une nouvelle vie dans une nouvelle ville, bien qu'inconnue, leur offrait aussi l'occasion de se rendre « invisibles ».

Trois interviewés jouissant d'une visibilité locale relatent que leur parcours de vie a été touché par l'exil. L'un d'entre eux habitait déjà la ville de São Paulo et avait préparé son départ à l'étranger, en sachant que ses activités dans la sphère d'une double vie l'exposaient à la répression. Il raconte librement, bien que rapidement, cette expérience. Il explique que son retour au Brésil se fait encore pendant le régime militaire, malgré l'instabilité sociale et les risques personnels, parce qu'il ne voulait pas «devenir allemand » et car il souhaitait se défaire du sentiment d'avoir eu un «parcours interrompu». Une autre interviewée s'exprime davantage sur les exils successifs auxquels elle et sa famille ont été soumis dans différentes villes brésiliennes en quête d'invisibilité sociale obtenue enfin à São Paulo par l'abandon de tout comportement soupçonneux à son égard. Un dernier interviewé ne fait que mentionner son exil quand il a été poussé à préciser certaines informations.

Dans les trois cas, on perçoit clairement un sentiment de tristesse vis-à-vis du départ du lieu d'origine. Par ailleurs, même si ces acteurs sociaux réussissent leur vie personnelle et professionnelle à São Paulo, les récits de l'impossibilité de reprendre leur ancienne vie dans leurs anciennes villes sont empreints de mélancolie. On exprime des sentiments de gratitude envers la camaraderie trouvée dans la ville d'accueil, en même temps qu'on regrette les anciennes occasions perdues : «Nous ne connaissions plus personne. C'était une autre époque ».

En analysant les différents discours relatifs à l'exil, on peut comprendre que l'exil à l'étranger est une issue reconnue et acceptée par les intellectuels brésiliens. Les interviewés, eux-mêmes membres de cette communauté en parlent à plusieurs reprises. Leurs propos constituent régulièrement des preuves de la reconnaissance à la fois des efforts des confrères universitaires dans la lutte contre la dictature militaire et des préjudices subis. Toutefois, le fait d'abandonner une ville brésilienne pour s'installer dans une autre et fuir des restrictions paraît ne pas bénéficier de la même reconnaissance.

Le sens même du mot «exil », plus facilement considéré comme un déplacement d'un pays à un autre, n'est pas la seule raison de cette différence d'appréciation. Il convient ici d'opposer la notoriété sociale à l'invisibilité sociale. Les intellectuels brésiliens ayant opté pour l'exil à l'étranger paraissent avoir embrassé la notoriété. Ayant une carrière professionnelle bien établie, ils en font usage, semble-t-il, pour pouvoir continuer à développer leurs travaux. En revanche, les intellectuels les plus jeunes paraissent embrasser l'invisibilité sociale, afin de pouvoir faire de même en sol brésilien. 


\subsection{En dépit des intérêts communautaires, vive le profit personnel!}

Face au contrôle idéologique du gouvernement militaire, certains universitaires choisissent d'en tirer parti.

Durant les premières années de l'instauration du gouvernement militaire mais aussi lors des entretiens, est dénoncée la mise à profit personnel de certains mécanismes de contrôle du gouvernement militaire. Les associations, en l'occurrence l'Association d'enseignants de l'Université de São Paulo - l'ADUSP (Associação 2004), certains intellectuels et neuf des interviewés font usage de leurs écrits et de leurs récits pour faire valoir un propos similaire : la dénonciation des méfaits, cette fois des collaborateurs et/ou des sympathisants de ce gouvernement. Alors que les faits sont énoncés, l'identité des agents est, dans la plupart des cas - hormis ceux publiquement connus - gardée confidentielle.

D'après ce qui en est dit, la conjoncture sociale et universitaire permettait le fait qu'un individu mette ses propres intérêts au-dessus des intérêts communautaires. Une des motivations de l'adoption d'un tel comportement dérive de la volonté de résoudre des divergences intellectuelles et du désir d'occuper des postes dans la haute administration universitaire. Il ne s'agit pas de «fermer les yeux » face à la répression ni de passer ouvertement pour un sympathisant. Ici, certains universitaires, notamment les intellectuels de droite, indiquent les interviewés, font appel à la répression militaire et établissent avec elle une alliance tacite.

Leurs récits ne désignent pas qu'un alignement sur les politiques du gouvernement militaire soit perçu comme une stratégie de protection ou même comme le fruit de la peur engendrée par le climat délétère que les interviewés relatent eux-mêmes. Il paraît même inconcevable pour cette partie de la communauté universitaire qu'un alignement sur ce gouvernement soit simplement le fruit d'une identification individuelle avec les principes, les actions et les politiques de ce gouvernement. Comment l'un d'entre eux pourrait-il partager des idées similaires avec une dictature militaire, avec leur ennemi annoncé ? - fera remarquer une interviewée.

Même si le mot «trahison » n'est pas employé pour caractériser ni cette période ni les actes de ces individus, les récits des interviewés laissent entrevoir un tel ressentiment. Une partie de la communauté universitaire a trahi l'autre, et ce pour des raisons vénales et carriéristes. Des «bandits » et des « héros » sont apparus, comme le décrit un interviewé, mais la communauté universitaire ne pourra «jamais pardonner » un tel comportement, complète une autre.

\subsection{La vie continue comme si de rien n'était}

L'indifférence quant à la conjoncture sociopolitique parait représenter un type de parcours de vie qui ne mérite pas l'attention de la communauté universitaire, plus 
particulièrement chez les interviewés. Aucun d'entre eux ne fait allusion à un tel type de comportement. Ils en parlent en ce qui concerne la population générale ; et ce, soit parce qu'elle méconnaissait la situation réelle du pays, soit parce qu'elle se fiait à l'illusion d'un avenir meilleur. Et, pourtant, ce type de comportement était bien réel, même chez les universitaires et chez les interviewés. Les individus, qu'ils aient été des universitaires ou tout simplement des brésiliens, n'ont pas tous eu un mot à dire au sujet du changement de système politique.

\section{La mémoire universitaire : un récit de lutte et d'opposition à la dictature militaire ?}

Une analyse des récits et de la bibliographie disponible montre que la fin de la décennie 1970 et le processus d' « ouverture politique » semblent réunifier la plupart de ces positions prétendument antagonistes. Ceux qui ont mené une double vie, qui se sont soumis à une autocensure ou qui ont défié la dictature dans le cadre de leurs travaux universitaires contestent ouvertement le gouvernement militaire.

Les récriminations quittent l'espace restreint des salles de cours. Les professeurs manifestent publiquement contre les actions du gouvernement militaire lors des réunions annuelles des scientifiques, dans le cadre de leurs publications, par la reprise d'anciennes associations ou la création de nouvelles. La communauté universitaire, en l'occurrence celle de l'USP, fait appel aux dénonciations publiques par moyen de presse et au sein de l'Assemblée des députés, pour restituer les postes aux collègues atteints par le gouvernement militaire.

Selon un interviewé, à partir de ce moment-là, s'investir dans la vie politique se fait naturellement. Certains membres de cette communauté intègrent la vie politique en coulisses, de manière à participer à la conjoncture politique et à sa reconstruction, et plus particulièrement pour faire changer le système éducatif brésilien. Plus tard, d'autres choisiront simplement de quitter la scène universitaire pour la scène nationale et deviendront même des personnalités politiques. Pour citer l'un des cas les plus célèbres, Fernando Henrique Cardoso, ancien professeur de l'USP destitué par acte gouvernemental et exilé à l'étranger, est élu président de la République l'année 1994 lors de la deuxième élection au suffrage direct, puis est réélu pour un second mandat.

Parmi les types de parcours susmentionnés, les interviewés - et la communauté universitaire d'une manière générale - semble s'identifier seulement avec trois d'entre eux, et plus particulièrement avec ceux mettant en évidence, d'une part, un comportement de refus des actions du gouvernement militaire et, d'autre part, la répression subie durant cette période de l'Histoire brésilienne.

L'analyse de leurs récits permet de dévoiler que, si le gouvernement militaire a établi la corruption, la subversion et notamment le communisme comme les ennemis de l'Etat brésilien, la communauté universitaire à son tour a institué la dictature mili- 
taire ainsi que ses sympathisants et ses collaborateurs comme ses ennemis et comme ceux de l'université. Une scission s'établit. Une scission mandatée par le gouvernement militaire, selon ce que racontent certains interviewés. Une scission qui dès lors est maintenue et soutenue après la fin de la dictature militaire. D'un côté, se trouvent les universitaires résistants et l'université elle aussi indubitablement résistante et engagée dans la résolution des problèmes nationaux, la déposition du gouvernement militaire incluse. De l'autre côté, figurent les militaires et leurs collaborateurs.

La communauté universitaire, en tant que groupe social, semble ainsi souhaiter élaborer une «mémoire commune, une mémoire partagée » (Candau 2005 : 71), ou bien une mémoire officielle, où l'on oublie les divergences propres aux contextes et aux relations sociales. On oublie une image et on en construit une autre de manière à dépeindre ou à feindre une histoire (Ricœur 2000). Les enjeux sociohistoriques fournissent la matière pour la création d'une «mémoire universitaire officielle » d'une université résistante.

Les anciennes divergences ainsi que les rivalités antérieures au régime militaire, citées quelquefois par la communauté universitaire, hier comme aujourd'hui, sont ré-signifiées en faveur d'une certaine représentation du passé. Les intérêts conservateurs sont interprétés comme une adhésion à l'ancien gouvernement militaire. Les sympathisants et les collaborateurs du régime militaire sont identifiés comme des membres de cet ancien régime. "Ils ne sont plus des nôtres » - aurait pu dire la communauté universitaire. Ils perdent ainsi leur statut d'universitaires et leur appartenance à cette communauté. Ils deviennent « les autres » avec qui on ne peut pas partager les mêmes valeurs. Corrompus par des propositions vénales et carriéristes, ils ne sont évidemment plus des universitaires. Ennemie de l'Etat, la corruption devient elle aussi l'ennemie de la communauté universitaire. Il ne s'agit pas, ici, de juger un comportement en fonction de son allégeance ou de sa contribution aux actions du gouvernement militaire ; il s'agit plutôt d'indiquer comment la communauté universitaire semble l'avoir signifié.

Les décisions conservatrices, notamment dans le cadre de la réforme universitaire de 1968, sont prises par certains comme un alignement sur le gouvernement militaire. Pour d'autres, les conservateurs ont en effet fait appel aux mécanismes de contrôle du gouvernement pour faire passer des résolutions allant dans leur intérêt, essentiellement pour en tirer des profits personnels. La «mémoire universitaire officielle » paraît avoir oublié que les forces progressistes et conservatrices se sont toujours opposées et que c'est seulement en de rares occasions que les progressistes en sont sortis « vainqueurs».

Il est aussi difficile pour la communauté universitaire d'accepter qu'elle n'ait pas toujours tenu des comportements compatibles avec la résistance au gouvernement militaire. L'indifférence ou l'ignorance face aux évènements sociopolitiques sont des comportements réservés à la population en général. Comment la communauté universitaire pourrait-elle annoncer ne pas être au courant de la «triste» et «diffi- 
cile » conjoncture sociopolitique, si l'université était l' «épicentre des évènements », comme le signalent certains interviewés ? Comment pourrait-elle utiliser une telle excuse ? Impossible. Une fois les autres opposants au gouvernement anéantis par la répression, l'université, épargnée par la violence dictatoriale, avait pris la responsabilité d'organiser la lutte contre le gouvernement militaire, déclare un interviewé. En toute connaissance de cause, comment la communauté universitaire pourrait-elle faire preuve d'indifférence face aux méfaits dictatoriaux ? Également impossible, rajoute une autre interviewée. La «mémoire universitaire officielle» défend que l'université a tenu son rôle de faire face à la dictature militaire, que ce soit par la résistance intellectuelle ou par une double vie partiellement consacrée à la clandestinité.

Face au silence entourant les actes de répression et de contrôle idéologique sur l'université, les interviewés relatent qu'ils se sont positionnés, de manière à les briser et à rompre avec la conjoncture sociopolitique instaurée par le régime militaire, et ce, malgré les dangers bien réels. Malgré le fait de vivre «indiscutablement une dictature », les universitaires ont agi selon une « liberté assumée », une «liberté réellement exercée », explique un interviewé. Une prise de risque consciente.

Dans cette optique, les interviewés relatent les actes de bravoure de la communauté universitaire. La résistance intellectuelle, qu'elle se fasse par la défiance intellectuelle ou par l'autocensure, vise, d'après les récits, à préserver l'université et à la restituer intègre à la société une fois la dictature militaire finie. Or, défiance intellectuelle et autocensure ne jouissent pourtant pas du même statut ni de la même appréciation. Le fait d'être pris comme un acteur ou, au contraire, comme un témoin oculaire des évènements sociopolitiques paraît jouer un rôle. Adopter un comportement d'autocensure pourrait éventuellement être associé à un comportement d'indifférence en ce qui concerne les évènements nationaux de l'époque, ou même à une possible collaboration avec la dictature militaire. À des comportements différenciés, vingt ou quarante ans après coup, correspondent des valorisations quasi antagonistes, même si l'objectif visé - «sauver l'exception universitaire »-a été le même.

L'adoption d'une double vie, et plus particulièrement celle menée dans la sphère de la clandestinité, met en avant la lutte des universitaires face aux méfaits dictatoriaux. Il fallait contourner les mécanismes de contrôle du gouvernement militaire pour le mettre à mal. Bien que vivre une double vie dans le champ de la clandestinité inspire de la fierté, la lutte armée ne jouit pas du même statut. La communauté universitaire paraît ne pas vouloir être identifiée avec un tel choix. Pourquoi ?

Ces deux types de parcours mettent en évidence une opposition formelle et une lutte, bien que dissimulée, contre le gouvernement militaire. Cependant, une «mémoire universitaire officielle» impliquant la lutte armée aurait pu évoquer l'existence d'un projet révolutionnaire à l'image d'une dictature de gauche, comme l'analyse Cordeiro (2009) en ce qui concerne la société en général. D'autre part, au- 
jourd'hui, les comportements associés à la clandestinité, tels qu'une affiliation à un parti politique clandestin et la production et la divulgation de publications censurées n'enfreignent plus le code juridique brésilien. Même les activités douteuses, telles que le transport de colis au contenu secret, toutes ces actions évoquées par les interviewés, restent acceptables et surtout légales à l'heure actuelle. Tous ces actes sont même vus comme des comportements de bravoure compte tenu de la période historique.

D'autre part, les récits dénonçant la souffrance des universitaires viennent compléter le cadre de cette «mémoire universitaire » et finissent en fait par mettre en valeur les attitudes de défiance. Pour avoir adopté un comportement de résistance, évoquent les interviewées, des universitaires se sont retrouvés au centre de la répression. Ils ont été mis en prison et, parfois, torturés. Certains de ses membres ont été destitués de leur poste et ont dû rétrograder professionnellement pour s'en sortir. D'autres ont été obligés de s'exiler. D'autres encore ont trouvé la mort ou sont restés à jamais marqués par les méfaits du gouvernement militaire. Certains d'entre eux, exilés, persécutés, assassinés, deviennent des symboles d'un parcours interrompu et empêché, voire gâché par la dictature militaire. Pourtant, les interviewés déclarent que, malgré tous les méfaits dictatoriaux, les universitaires sont parvenus à réussir leur vie, tant privée que professionnelle.

Un tel récit, qui oppose les faits des universitaires et les méfaits de la dictature, peut être utilisé pour exiger une réécriture du discours historique, social et politique en concordance avec les attentes d'un groupe social ${ }^{3}$. On cherche ainsi à défaire les actes du gouvernement militaire qui limitaient ou qui brisaient l'autonomie universitaire. On essaie aussi de construire une seule et unique "mémoire universitaire », plus forte, plus visible pouvant faire passer des politiques compensatoires et obtenir la reconnaissance des efforts entrepris durant ces années de lutte et de perte au niveau personnel, professionnel et institutionnel. C'est justement en faveur de la construction d'une «mémoire universitaire » unique, semble-t-il, que la collaboration de certains avec le gouvernement militaire est révélée, alors que l'identité des individus reste secrète. On peut donc analyser ce type de discours comme une tentative de reconquérir un «quotidien » qui leur a été volé, de reconstituer des «parcours interrompus », de récompenser la "génération sacrifiée » et d'exercer leurs droits civiques sur le sol brésilien.

\footnotetext{
3 Pour une discussion sur l'usage politique et identitaire des mémoires et des discours sociaux, voir Benbassa (2010) et Michel (2010).
} 


\section{Conclusion}

La dictature militaire marque une période assez récente de l'histoire du Brésil. Quoique jamais véritablement atteignable, il paraît difficile de trouver la «juste » distance d'analyse par rapport aux faits et aux évènements de cette période ; et ce, tout autant pour la personne qui tient la plume en ce moment.

Après la remise des pouvoirs aux civils, la scène universitaire, sociale et politique voit des comportements, maintes fois, (quasi-) similaires à ceux de l'époque militaire. Certains individus luttent pour que les actes du gouvernement militaire soient examinés, jugés et sanctionnés. D'autres craignent le retour des militaires au pouvoir et préfèrent reléguer au silence leurs opinions et toute discussion à caractère politique. D'autres encore désirent en silence le retour du même gouvernement militaire.

Malgré cette diversité de comportements, chez les universitaires, un discours plutôt consensuel se répand. On voit une «mémoire universitaire » évoquant la figure d'une université résistante et défiante des politiques et des actes militaires. L'image d'une dictature qui a taché le quotidien et l'histoire de l'université et de la société brésiliennes perdure.

Quoi qu'il en soit, l'université et la société poursuivent leur évolution. Comme toujours, leur avenir continue à être influencé par de multiples facteurs, par les actes de différents acteurs sociaux, par le passé et par les enjeux contemporains. Une nouvelle époque est en train de s'écrire.

\section{Bibliographie}

Associaçao dos Docentes da USP (2004). O controle ideológico na USP: 19641978. São Paulo : ADUSP.

Benbassa E. (2010). «Juste mémoire » ou raisonnable oubli (Préface).Gouverner les mémoires: les politiques mémorielles en France / ed. by J. Michel. Paris: Presses Universitaires de France (PUF), pp. IX-XV.

Candau J. (2005). Anthropologie de la mémoire. Paris: Armand Colin Editeur.

Coggiola O. (2001). Governos militares na América Latina. São Paulo: Contexto.

Cordeiro J. M. (2009). Anos de chumbo ou anos de ouro ? A memória social sobre o governo Médici. Estudos Históricos, vol. 22, nº 43, p. 85-104.

Ditaduras militares na América Latina (2004) / ed. by C. Wasserman \& C. A. B. Guazzelli. Porto Alegre: Editora da URFGS.

Fausto B. (2001). História do Brasil. São Paulo: EDUSP. 